\title{
Lightness depends on immediately prior experience
}

\author{
VIDAL ANNAN, JR. \\ Rutgers University, Piscataway, New Jersey \\ and \\ ALAN GILCHRIST \\ Rutgers University, Newark, New Jersey
}

\begin{abstract}
The lightness hangover illusion is an unusually robust, long-lasting, prior-experience-based lightness effect. The effect occurs in the Mondrian world, a miniature chamber with interior walls covered with dark gray to black patches. The lightest patch in this scene, physically dark gray, looks white. When real whites and light grays are added to the scene, all the patches darken, but at an unusually slow rate. For several seconds, the white patches look self-luminous and the other patches continue to look very light. The luminosity fades and the other patches darken only after $2 \mathrm{~min}$. We tested three possible explanations for this illusion: retinal adaptation, lightness persistence, and anchor persistence. The results clearly support anchor persistence, which is caused by the presence of steady patches, surfaces that retain their luminance values across scenes. The data also show that the size of the illusion varies directly with the number of these steady patches.
\end{abstract}

Does lightness depend on prior experience?

Despite a lack of empirical support, theories of lightness, or perceived surface gray level, have reserved a prominent place for some form of past experience. Helmholtz (1866/1962), for example, attributed lightness constancy (perceiving a surface as constant in lightness despite changes in illumination level) to a process of learning:

We have abundant opportunity to examine the same object colors in full sunlight, in the blue light from the clear sky, the weak white light of the clouded sky, and the reddish yellow light of the sinking sun or of candlelight - not to mention the colored reflections from surrounding objects. Seeing the same objects under these same illuminations, we learn to get a correct idea of the object colors in spite of differences of illumination. We learn to judge how such an object would look in white light...

Hering, another pioneer in lightness research, proposed memory color (1874/1964), a cognitive mechanism that improves lightness constancy when an object with a characteristic shape and color (e.g., a banana) is recognized as familiar. According to Hering, the shape and color of an object are associated in memory in such a way that when the object is later encountered under different conditions of illumination, its perceived color

Correspondence should be addressed to V. Annan, Jr., Center for Cognitive Science, Rutgers University, 152 Frelinghuysen Rd., Piscataway, NJ 08854-8020 (e-mail: avidal@ruccs.rutgers.edu).

Note-This article was accepted by the previous editorial team, headed by Neil Macmillan. shifts toward the associated color. He wrote, "The color in which an object appears, disregarding the limiting cases, acquires a certain stability and becomes in our memory an enduring, integrated constituent part of the object" (Hering, 1874/1964, p. 20).

Hering's theory includes another factor, retinal adaptation, that also involves prior experience. When the illumination increases, the luminance emitted from an object also increases. But according to Hering, the brighter overall visual field causes a reduction in sensitivity of the retinal receptors, bringing the receptor response down toward its level in the original illumination. Kreis (1905) also provided one of the earliest experiments and discussions on retinal adaptation, particularly in the chromatic domain.

According to Hurvich and Jameson (1966), their opponent processing model of lightness accounts merely for the raw brightness or darkness of retinal areas. Whether this brightness or darkness is perceived as properties of the surfaces or as properties of the illumination depends on the "interpretation of the total situation" (p. 88). This interpretation is presumably the product of learning.

But despite the theoretical role of past experience, there has been little, if any, empirical support. For example, if learning is involved, then one could assume that the more developed (or evolved) of a species should display better lightness processing ability. But experimental results are to the contrary; infants and animals do not show less constancy than adult humans do (Burzlaff, 1931; Köhler, 1917). Studies of memory color have revealed either no effects or very small effects, and these occur only under conditions in which the stimulus color 
is impoverished or unstable (Bruner, Postman, \& Rodrigues, 1951; Duncker, 1939; Hanawalt \& Post, 1942; Zuckerman \& Rock, 1957). Thus, Bolles, Hulicka, and Hanly (1959) suggested that memory color effects may represent no more than a response bias. As for adaptation, although it has been shown to influence the perceived brightness or dimness of a surface (Davis \& Brooks, 1977; Over, Broerse, Crassini, \& Lovegrove, 1974), there is no empirical evidence showing an effect on lightness, the intrinsic gray shade of a surface.

In addition, the Gelb (1929) effect has been cited as evidence against prior experience. In this illusion, a black paper suspended in midair appears white when it is the only surface illuminated by a spotlight. When a real white paper is also brought into the spotlight, the original black paper suddenly appears much darker. But when the white paper is removed and the original stimulus conditions are restored, the black paper quickly appears white once again, despite the prior experience of the observer.

But just as theories that invoke prior experience lack empirical support, there is also empirical evidence for prior experience that lacks a theoretical interpretation. For example, with respect to the Gelb effect, Newson (1958) showed that when the real white surface is subsequently removed from the spotlight, the black paper takes as long as $7 \mathrm{sec}$ to return to white. In addition, observers who experienced the repeated addition and removal of the white surface reported that the black paper did not look as white as it originally appeared.

Cataliotti and Bonato (2003) have reported a similar effect. They show that, although a disk surrounded by darkness normally looks white, it looks gray when preceded by another disk of higher luminance.

Among the observations that Wallach (1948) made concerning his disk/annulus stimulus, he noted an asymmetrical effect of adding or removing the annulus. When the annulus is added, disk lightness changes immediately to reflect the new luminance ratio. When the annulus is removed, however, disk lightness changes much more slowly, taking a few seconds to fully lighten.

Gilchrist (1980) reported a hysteresis effect in his experiments on depth and lightness. When the same observer viewed a target successively in two alternative depth organizations, the lightness difference between these displays was approximately half that found when separate observers viewed the two displays. This implies that the lightness assigned to the target in the first depth organization persists into the second.

Another study, conducted by Shimozaki, Eckstein, and Thomas (1999), addressed the question of how the lightness of a specific object is represented when its luminance changes over time. Using a Ternus-type two-frame apparent motion illusion (Ternus, 1938), they found that when the luminance of a target changes as it moves, the lightness of the surface at Time 2 tends toward its lightness at Time 1 .

Taken together, these empirical findings share several aspects, a careful analysis of which can help us sort out the types of past experience that may affect lightness and that may not. First, in all cases the lightness illusion seems to depend on events that occur in the very recent past. This potentially rules out knowledge and learning, as proposed by Helmholtz and Hering, as explanations for these illusions. Helmholtz's learning idea, for example, involves extended periods in which information is acquired about the relationships among surfaces, illumination, and luminance. Therefore, it does not seem applicable to illusions that occur immediately. Nor can memory color account for these examples if the surfaces used have generic shapes that lack a characteristic color. As for retinal adaptation, either it is controlled for in most experiments (Cataliotti \& Bonato, 2003) or the experimental results run opposite to a retinal adaptation prediction (Shimozaki et al., 1999).

We sought to study these effects of immediately prior visual experience using a robust and long-lasting illusion observed by Cataliotti and Gilchrist (1995). This illusion occurs when one views a miniature chamber they called the Mondrian world (Figure 1).

\section{The Lightness Hangover Illusion}

The interior of the Mondrian world is entirely covered with a collage composed of dark gray to black rectilinear surfaces. The observer's head is placed inside this chamber so that his/her visual field is completely filled by the interior walls of the chamber. Observers report that the highest luminance surface in the scene, physically dark gray, looks white, in accord with the highestluminance-as-white anchoring rule (Gilchrist et al., 1999). All the other surfaces are seen in relation to the highest luminance. As the observer continues to look, a number of real white, light gray, and middle gray surfaces are added to the room by sliding the far wall (which is actually part of a longer sliding panel) to the right (Scene 2). The observer now reports both that the real white surfaces look self-luminous and that all the other surfaces appear darker than before. But surprisingly, the surfaces appear nowhere near as dark as they would without prior exposure to Scene 1. For example, the dark gray surface, which looked white in Scene 1, now appears light gray. This lightness hangover slowly decays over time, darkening over the course of a minute and more to the dark gray that it would appear as without prior exposure to Scene 1.

\section{EXPERIMENT 1 Replication}

Before testing any formal hypotheses, we replicated the findings of Cataliotti and Gilchrist (1995), paying special attention to the size and temporal duration of the illusion.

\section{Method}

Subjects. Seventy-two Rutgers University students volunteered for the study. These subjects, as well as the subjects in all subsequent experiments, had normal or corrected-to-normal vision and were completely naive to the purpose of the experiment. 

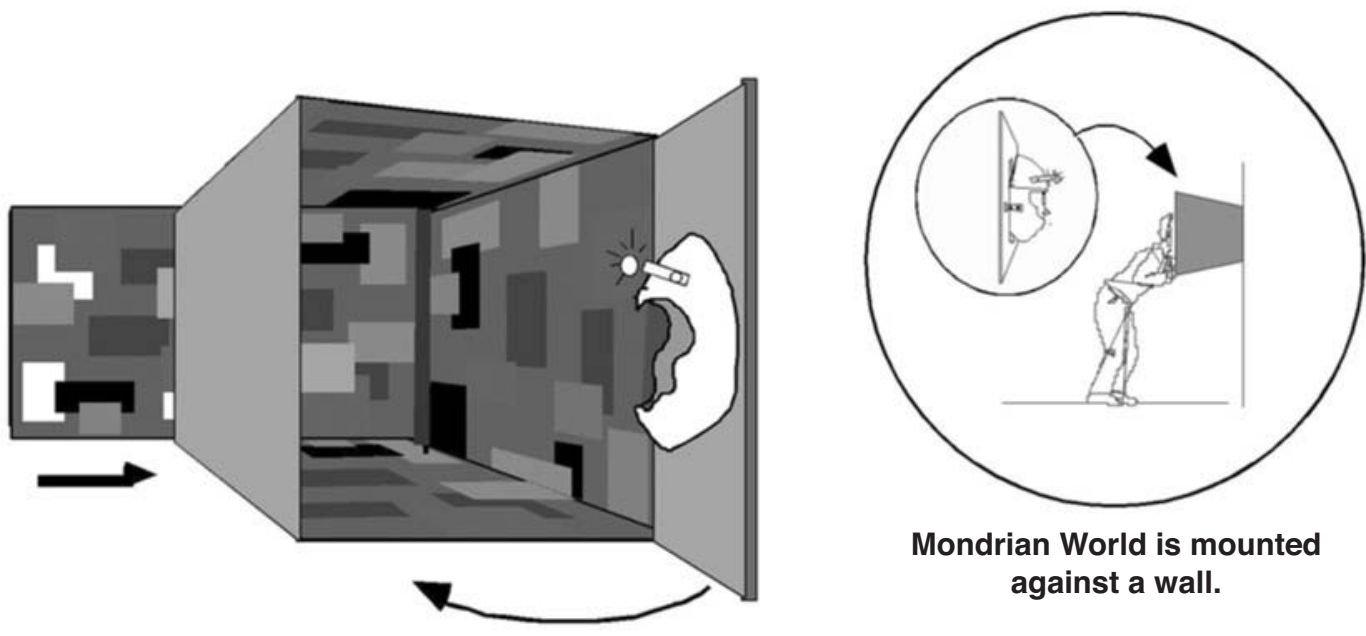

Figure 1. The Mondrian world.

Apparatus and Stimuli. The Mondrian world, illustrated in Figure 2 , is a miniature, trapezoidal room constructed of plywood that is attached to a wall in the laboratory so that its bottom edge is $110 \mathrm{~cm}$ above the floor. The top, bottom, and sidewalls of the room are all trapezoidal panels, $36 \mathrm{~cm}$ on the short side and $69 \mathrm{~cm}$ on the longer side. The nonparallel edges are $50 \mathrm{~cm}$. The near wall of this room $(69 \times 68 \mathrm{~cm})$, which is at the wider end of the trapezoids, is a door that is hinged on the left side. The middle of the door contains an oval-shaped hole $(28 \times 20 \mathrm{~cm})$ fitted with a converted hockey mask that serves to position and stabilize the head as the observer looks into the chamber. Directly above the facemask, on the inside of the door and out of view of the observer, is a small $150-\mathrm{W}$ incandescent light bulb that illuminates the chamber. The distance from the near wall (door) to the far wall is $46 \mathrm{~cm}$. This far wall $(38 \times 36 \mathrm{~cm})$ is actually part of a longer sliding panel $(76 \times$ $36 \mathrm{~cm}$ ), half of which is out of view when one is looking through the mask. The section of the panel that is in view from inside the room can be replaced with the hidden section by sliding the whole panel to the right. The inside walls of the Mondrian world (excluding the door panel) are completely covered by a collage of rectilinear patches ranging in Munsell value from 4.5 (dark gray, luminance $103.5 \mathrm{~cd} / \mathrm{m}^{2}$ ) to 2.0 (black, $18.9 \mathrm{~cd} / \mathrm{m}^{2}$ ). Some of these patches are painted with matte paint, but the majority are actual gray patches of papers cemented in place. The patches, which number about 90 , are evenly distributed on the top, bottom, and sidewalls and range in surface area from approximately $40 \mathrm{~cm}^{2}$ to $300 \mathrm{~cm}^{2}$. The sliding panel in view from inside the room is patterned with about 35 patches of actual gray papers that also range from Munsell 4.5 to 2.0. The section of the sliding panel that is out of view is patterned with approximately 35 white to black papers that range from Munsell 9.5 (white, $644 \mathrm{~cd} / \mathrm{m}^{2}$ ) to Munsell 2.0 (black, $18.6 \mathrm{~cd} / \mathrm{m}^{2}$ ).

Procedure. Before the observer looked in the Mondrian world, he/she received the following instructions:

You are about to participate in an experiment in which you will have to make gray shade matches for particular surfaces that you will see in this room (point to the Mondrian world). When you look in, you will see that the walls of the room are covered with several different gray surfaces. I want you to spend a few seconds looking all around and then I am going to ask you to find a specific surface in the scene. When you find this surface, I want you to try to get a good impression of its gray shade and tell me if it looks white, light gray, middle gray, dark gray, or black [the order of presentation of this list was randomized for each subject]. After you have made this match, I will slide in several new surfaces into the scene. Look around for a few seconds; then I will ask you to find a new target. When you find this target, I again want you to try to get a good impression of the gray shade of the target and try to remember it. When I turn out the lights in the room, I want you to remove your head from the chamber and look at this Munsell chart (positioned on a table directly behind the observer). As quickly as you can,
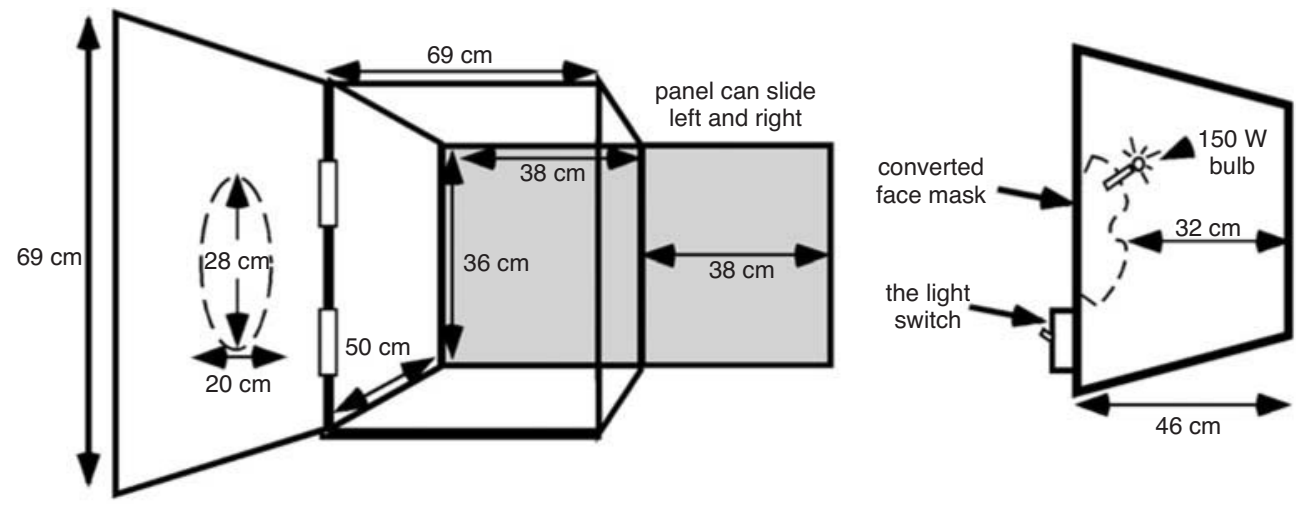

Figure 2. A schematic rendering of the Mondrian world with dimensions. 
I want you to select the chip that matches the gray shade of the target. After this, you will also select for me the chip that matches the gray shade of the first target.

In the instructions, more emphasis was placed on remembering the target in Scene 2, because it was there that the illusion occurred. The target in Scene 1 was given less importance because pilot studies showed very little variability in the matches.

The observer looked into the chamber and was asked to scan the scene for a few seconds and then find the star-shaped target. The observer then gave a qualitative lightness match for the target.

The experimenter then moved the sliding panel to the right, introducing the new scene. The observer had 5, 20, 80, or $160 \mathrm{sec}$ to look around, find another star-shaped target, and note its gray shade.

The light in the Mondrian chamber was then turned off. At this signal, the observer withdrew from the room, turned to the Munsell chart and quickly made a match for the second target and then for the first target.

In the control condition, the observer viewed only Scene 2 for $5 \mathrm{sec}$ without prior exposure to Scene 1 and matched the target.

Design. Separate observers were used to avoid cross-trial experience effects, 15 in each of the four time interval conditions and 12 in the control condition.

\section{Results and Discussion}

The results are shown in Figure 3. The matches have all been converted from Munsell values to log reflectance values, and all statistical analyses are one tailed because a target will only get darker, never lighter, in the presence of a higher luminance.

There were no statistical differences for the matches for the Scene 1 target across all conditions; therefore, all values were collapsed together and represented as the far left bar in the graph. The bar to the far right in the graph represents the baseline, or the mean match for the Scene 2 target in the control condition. This value is significantly different from the target in Scene $1[t=18.21, p<.05]$.

The initial drop, to the value at $5 \mathrm{sec}$, is the result of the addition of the higher luminances to the scene. After that, the scene does not change and the drop is gradual. At the shortest viewing interval of $5 \mathrm{sec}$, the target appears light gray, which is significantly different from the target in Scene $1[t=7.6, p<.05]$, as well as from the baseline match $[t=2.77, p<.05]$. The graph also shows that the perceived lightness of the target in Scene 2 continued to drop but did not reach baseline even after $160 \mathrm{sec}$ of continuous viewing $[t=1.5, p>.05]$. Note that in this and all subsequent analyses, the error bars are calculated using a $95 \%$ confidence interval.

\section{The Hypotheses}

We next tested three possible explanations of the lightness hangover illusion: (1) retinal adaptation, (2) lightness persistence, and (3) anchor persistence.

\section{EXPERIMENT 2 Inserting a Darkness Interval}

Adaptation to the increase in average luminance from $22.8 \mathrm{~cd} / \mathrm{m}^{2}$ in Scene 1 to $56.5 \mathrm{~cd} / \mathrm{m}^{2}$ in Scene 2 could plausibly cause the surfaces in Scene 2 to initially look too light, and then slowly darken to baseline as the retina reached equilibrium. If so, then if we lowered the aver-

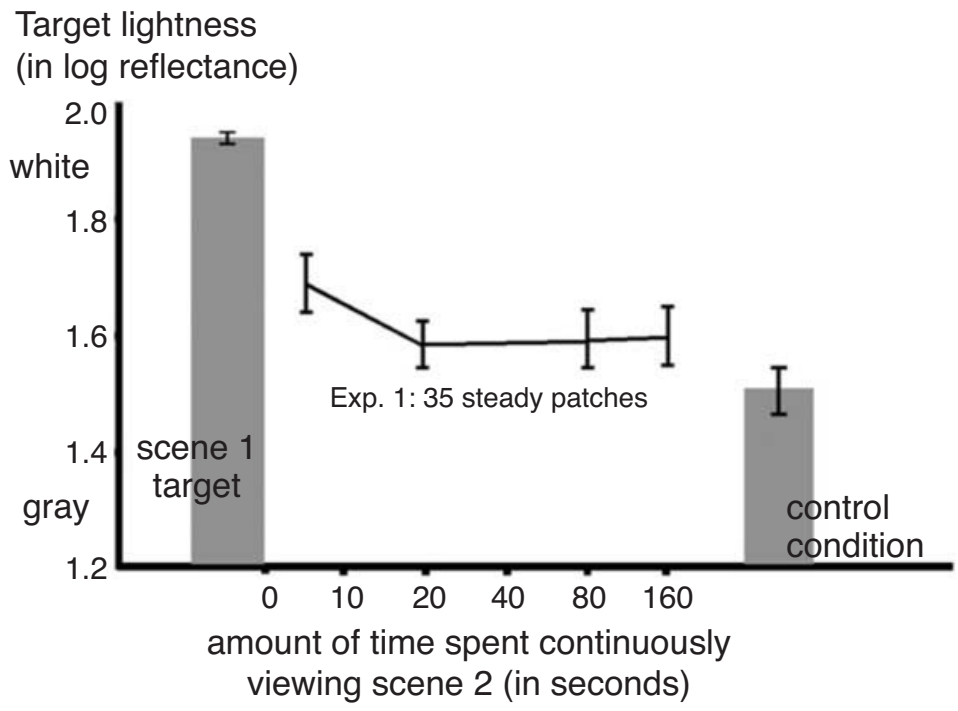

Figure 3. Results from Experiment 1, replication of Cataliotti and Gilchrist (1995). The far left bar represents the matches of the target in Scene 1. The far right bar represents the target in Scene 2 without prior exposure to Scene 1 (the control condition). The line represents the matches of the Scene 2 target after exposure to Scene 1 . The target initially appears light gray and slowly darkens. However, even after 160 msec of continuous viewing, it is still lighter than in the control condition. In this and all subsequent analyses, the error bars are calculated using a $95 \%$ confidence interval. 
age luminance immediately prior to Scene 2 even more, we should produce an even stronger temporal effect in Scene 2 . To test this, we switched off the illumination in the chamber while the sliding panel was being moved.

\section{Method}

This experiment was identical to the 5-sec condition in Experiment 1 except for the following changes: (1) An interval of 2-3 sec of darkness was inserted between the scenes by turning off the lights in the Mondrian world chamber. (2) There were 15 observers each in the experimental and control conditions.

\section{Results and Discussion}

The results of this experiment are shown in Figure 4. The bar to the far right, labeled the basic illusion, represents the standard hangover illusion at 5-sec. It will be used for comparison in subsequent graphs. Again the target in Scene 1 was perceived as white. The target in Scene 2, which followed the short period of darkness (the second bar from the left), was perceived to be middle gray, which is identical to baseline $[t=0.45, p>.05]$. These results do not show the lightening of the target in Scene 2 that is predicted by retinal adaptation. In fact the hangover illusion was completely eliminated. These results suggest that retinal adaptation does not explain the Mondrian world illusion. Indeed, the fact that the darkness interval completely destroyed the standard hangover illusion at $5 \mathrm{sec}$ itself requires explanation.

\section{Anchor Persistence}

How else might we explain the hangover illusion? We can get some clues by considering a real-world conditiona dining room in which the highest luminance is in- creased by raising the illumination level with a dimmer switch (rheostat). Normally when the illumination level in a room is raised, the highest luminance increases, as do the luminance values of all the other surfaces in the scene. All the surfaces are immediately reanchored using the newly established anchor (highest luminance). If this response to the increase in illumination were not immediate, surfaces would appear to become lighter and then gradually return to their original lightness values. But this is not what happens.

This dining room example illustrates that an increase in the illumination produces both an increase in the highest luminance and a dynamic increase in the luminance values of individual surfaces. It is possible that when both these changes occur simultaneously and in the same direction, the implication of an illumination change is stronger. In the dining room, the increase in the highest luminance and the luminance values of the surfaces occur simultaneously. This strongly implies a change in illumination level, and the surfaces are immediately reanchored.

On the other hand, in the Mondrian world the highest luminance increases, but all of the continuously visible patches remain constant in luminance. We hypothesized that whereas the change in highest luminance prompts reanchoring, the patches that retain their luminance, the steady patches as we will call them, indicate that the illumination has not changed, and thus the original anchor should not be updated. These conflicting cues slow down the overall anchoring process, producing the hangover illusion.

As in the familiar critique of Helmholtz's theory, it is not enough to suggest that illumination level is taken into

Target lightness

(in log reflectance)

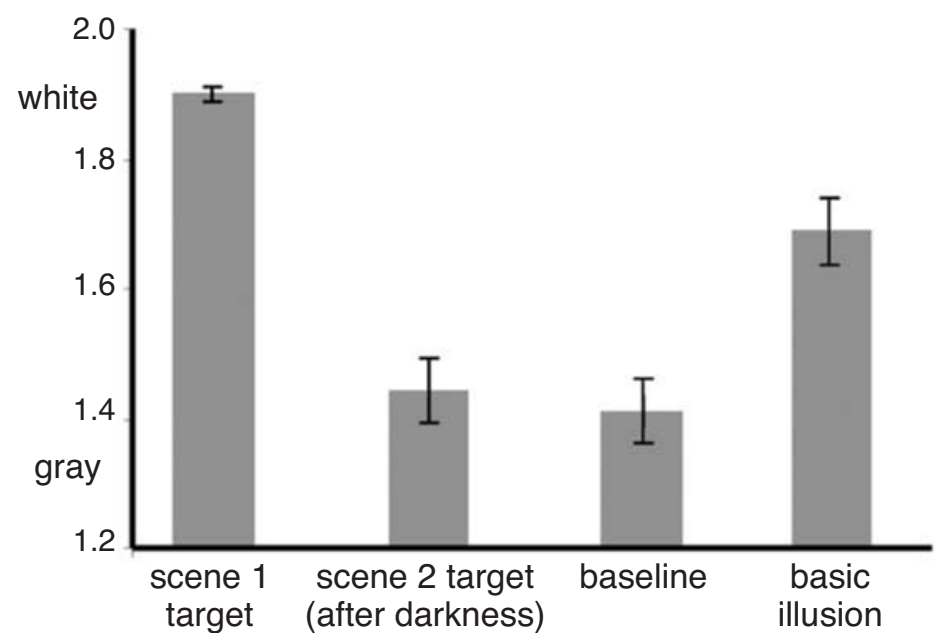

Figure 4. Results from Experiment 2, intervening period of darkness. As the graph shows, after the period of darkness, the target in Scene 2 appears as it would without prior exposure to Scene 1 (baseline). The bar to the far right represents the basic hangover illusion at $5 \mathrm{sec}$, or the expected size of the illusion without the period of darkness. 
account. One must specify concretely what stimulus variable represents the level of illumination. Likewise, here the question is what stimulus variables signal an increase in illumination level. We carried out a program of experiments to examine these issues.

\section{EXPERIMENT 3 \\ Eliminating the Steady Patches}

\section{Method}

If the hangover illusion is indeed caused by the presence of steady patches, it should be possible to destroy the illusion by eliminating them. To test this, we covered the top, bottom, and side panels with homogeneous black paper. If this did not completely eliminate the steady patches, at least their number was reduced from 35 to 4 . Apart from this change, Experiment 3 was identical to the 5-sec condition of Experiment 1. Ten observers served in each condition.

\section{Results and Discussion}

The results show (Figure 5) that the relative absence of steady patches wiped out the hangover illusion. The target in Scene 2 was perceived as middle gray, which was equivalent to the baseline value $[t=0.36, p>.05]$.

This outcome is consistent with an all-or-none role for steady patches. But it also might be that the size of the illusion depends, in a graded way, on the number of steady patches. If so, we should be able to obtain an even stronger illusion by increasing the number of steady patches (beyond that of Experiment 1).

\section{EXPERIMENT 4 Increasing the Number of Steady Patches}

In this experiment, we tested the effect of adding more steady patches in Scene 2. The number of steady patches in this experiment was increased from the 35 in Experiment 1 to 60 in Experiment 4.

\section{Method}

The method was identical to that of Experiment 1, except for the following changes.

For the transition from Scene 1 to Scene 2, the sliding panel was moved only half as far to the right, allowing only 15 new white and light gray patches to enter the scene. Twenty-five of the surfaces from Scene 1, including the target, remained in view; they just moved from the left visual field to the right. The number of white and light gray surfaces in Scene 2 was comparable to that in the previous experiments. The target from Scene 1 also served as the target in Scene 2.

Ten observers served in the 5 -sec condition, 13 in the 20 -sec condition, and 15 each in the $40-\mathrm{sec}, 160$-sec, and control conditions.

\section{Results and Discussion}

Figure 6 shows that at the shortest interval of $5 \mathrm{sec}$, the target was perceived to be a very light gray (mean log reflectance $=1.79$ ), significantly lighter than in Experiment $1[t=2.0, p<.05]$. After $160 \mathrm{sec}$ of continuous viewing, the target still had not darkened to baseline $[t=$ $4.42, p<.05]$. In fact, it still appeared as a very light gray.

These results suggest that the strength and duration of the hangover illusion varies with the number of steady patches, as if each steady patch "casts a vote" that the illumination has not changed and therefore the old anchor should remain in force.

So far, we had accumulated strong evidence to support the idea that the lightness hangover illusion is the result of the presence of steady patches in the scene. By maintaining their luminance values despite changes in the highest luminance, these surfaces indicate that the illumination level in the scene has not changed.

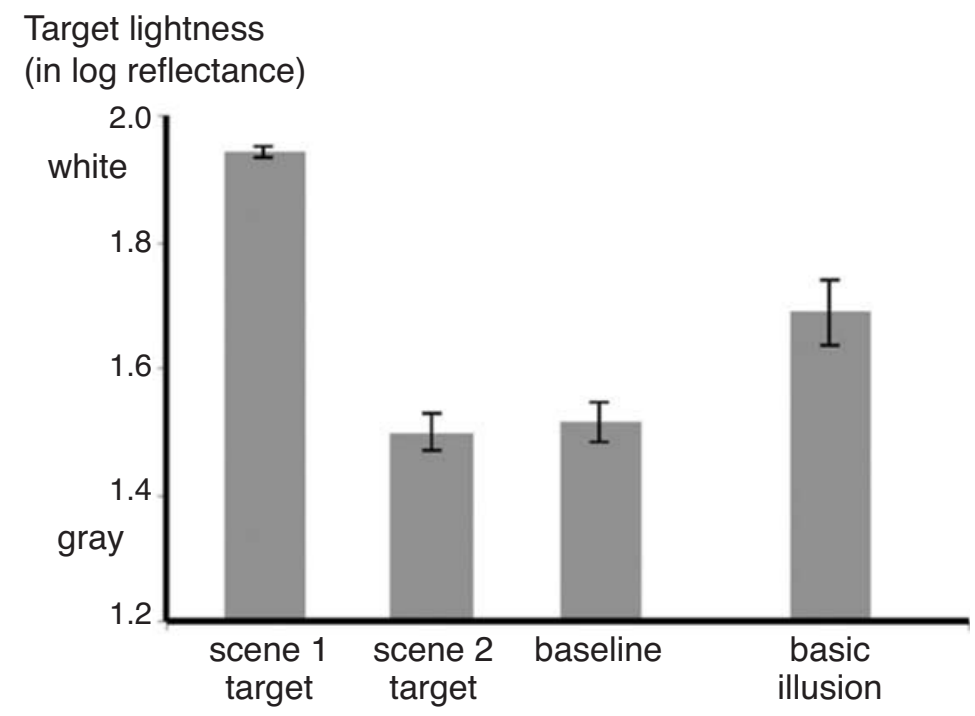

Figure 5. Results from Experiment 3, reduced number of steady patches. When the side panels are covered to reduce the number of steady patches, the illusion is eliminated. The graph shows that the target in Scene 2 is not significantly different from the baseline under these conditions. 


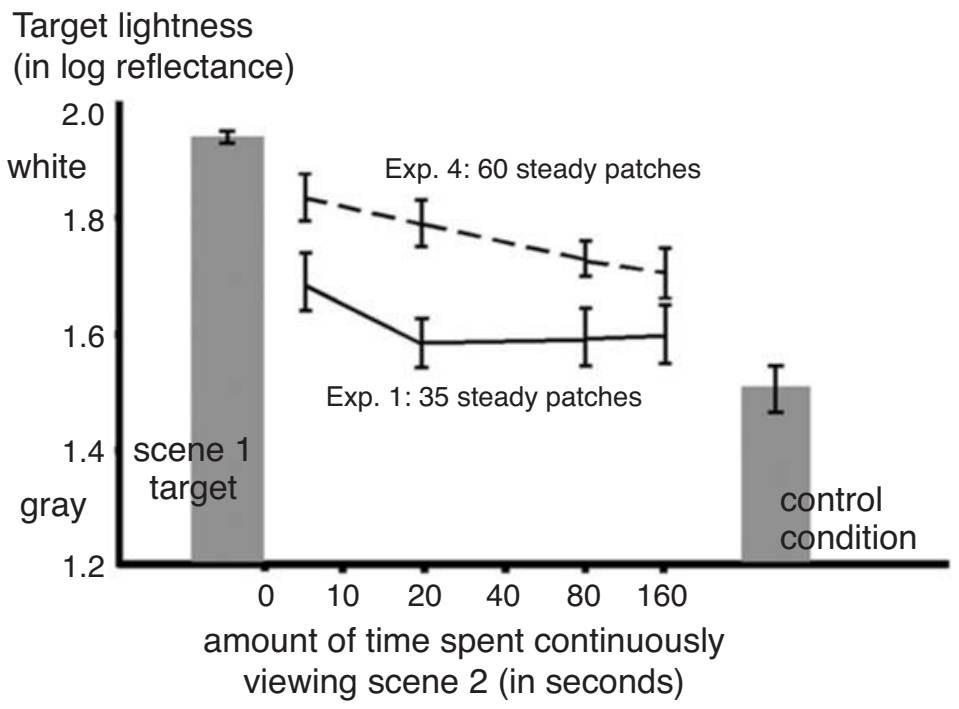

Figure 6. Results from Experiment 4, increased number of steady patches. The lightness hangover effect increased when more steady patches were maintained across scenes, as represented by the upper curve. The initial lightness was whiter than in the basic illusion (Experiment 1), and the target maintained this lightness value beyond 160 sec of continuous viewing.

\section{Lightness Persistence}

However, there was a confound in Experiment 4, because the target patch was continuously visible and its perceived identity over time was strong. It was therefore possible that all or part of its lightness hangover was due, not to persistence of the anchor as hypothesized, but to a persistence of target lightness. Research has shown, for example, that when a target surface moves to a new location and changes its luminance, its lightness at that time tends to be biased in the direction of the originally perceived lightness (Shimozaki et al., 1999). We call this process lightness persistence. Experiment 5 was conducted to tease these variables apart.

\section{EXPERIMENT 5}

\section{Anchor Persistence Versus Lightness Persistence}

In Experiment 5, we preserved the strong identity of the target while also reducing the number of steady patches. We covered the sidewalls and moved the sliding panel the full distance in such a way that no patch on the far wall from Scene 1 was present in Scene 2, in effect minimizing the number of steady patches. According to anchor persistence, this should destroy the illusion. But we also kept the target in view. According to lightness persistence, this strengthening of target identity should increase the size of the illusion.

\section{Method}

The method was identical to that of Experiment 3, except for the following changes.

In this experiment, the target was a separate surface. It was suspended from the ceiling of the Mondrian chamber but lay in the same plane as the sliding panel. When viewed through the viewing mask, the star appeared to be integrated into the sliding panel collage just as in the previous experiments. The sliding panel moved independently behind the star while the star remained stationary.

Twelve observers served in the experimental condition, and there were 10 in the control condition.

\section{Results and Discussion}

The results for this experiment are shown in Figure 7. In Scene 2, the target was matched as middle gray, which is statistically the same as the control condition $[t=$ $1.02, p>.05]$. The results show no lightening of the target as predicted by lightness persistence. Therefore, we can conclude that the illusion in Experiment 4 was not caused by lightness persistence.

\section{EXPERIMENT 6 Monitoring the Steady Patches}

The data so far suggest that the hangover illusion depends on the number of steady patches. This is clearly illustrated in Figure 8, which shows the strength of the hangover illusion that we had obtained in each of the experiments thus far, as a function of the number of steady patches.

However, to provide information against a change of illumination, the visual system must have continuous access to the luminance information from the steady patches during the change from Scene 1 to Scene 2. This does not mean that each patch must be actively attended. As long as it remains in the field of view, each patch can be passively monitored. If the luminance of a patch were to change, this change would immediately command atten- 


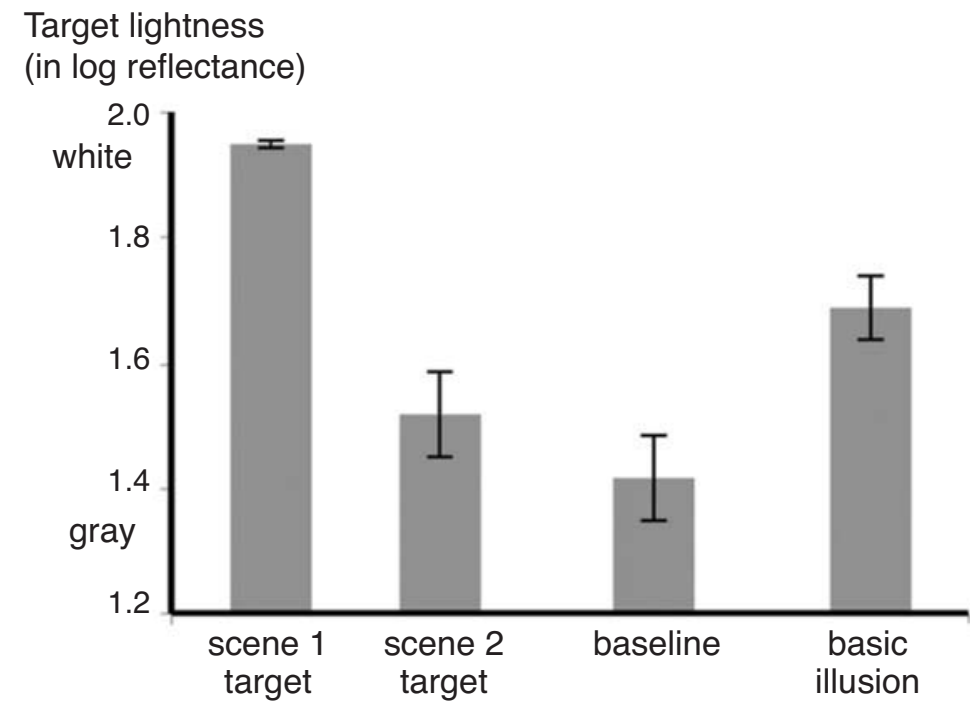

Figure 7. Results from Experiment 5, anchor persistence versus lightness persistence. The graph shows that when the white surfaces are added, the target in Scene 2 immediately darkens to baseline. This result supports anchor persistence.

tion (Klein, Kingstone, \& Pontefract, 1992). This analysis is consistent with the surprising results that we obtained in Experiment 2. Presumably the illusion was destroyed because the interval of darkness prevented continuous monitoring of the steady patches.

The number of passively monitored patches is important in this respect because the more of them there are in the scene, the greater the likelihood that at least one would shift in luminance if there was in fact a change in illumination. By not changing in luminance while being

\section{Target lightness after $5 \mathrm{sec}$}

(in log reflectance)

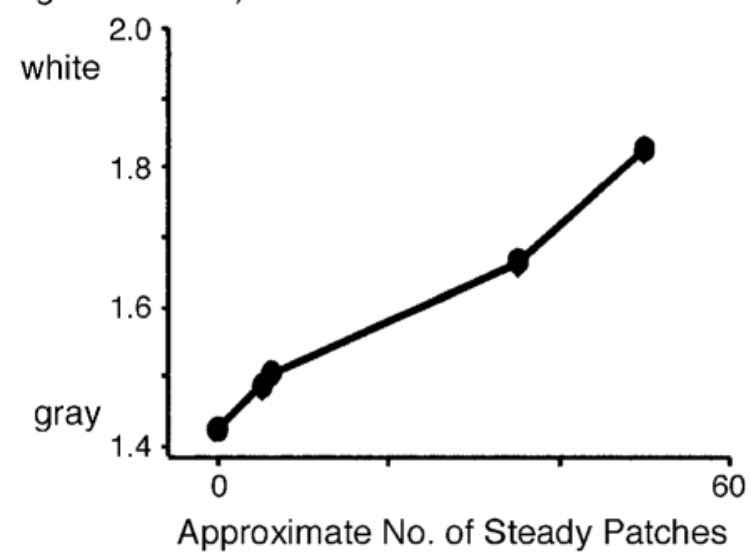

Figure 8. A comparison of results across all experiments. The points on the graph represent, from left to right, Experiment 2, Experiment 3, Experiment 6, Experiment 4, and Experiment 1. The graph clearly shows that there is a direct relationship between the number of steady patches in each experiment and the size of the lightness hangover illusion observed. passively monitored, each of the 35 or more steady patches can be said to "cast a vote" in favor of no change in the illumination.

In Experiment 6, the continuous monitoring of the steady patches was disrupted by having the observer remove his/her head from the Mondrian chamber between scenes. We hypothesized that this would make verification of the constant luminance of the steady patches more difficult, eliminating or reducing the hangover effect.

\section{Method}

This experiment was identical to the 5-sec condition of Experiment 1 , except that the observer's head was removed from the Mondrian chamber for about $2-3 \mathrm{sec}$ while the sliding panel was moved, during which time the observer looked around the well-lit laboratory. The observer then looked back into the chamber and had $5 \mathrm{sec}$ to find and match the target.

\section{Results and Discussion}

The results are shown in Figure 9. The hangover illusion is substantially reduced, but not completely eliminated. The lightness of the target $(M=1.57)$ is significantly darker than the basic illusion, but significantly lighter $[t=2.36, p<.05]$ than baseline. These results show that passive monitoring of the steady patches is important. But we cannot explain why the illusion was not completely eliminated, as it was by the darkness interval in Experiment 2. In fact, it is important to note that in a subsequent replication of this experiment, not reported here, the hangover effect was completely eliminated.

\section{GENERAL CONCLUSIONS}

We can conclude that our hangover illusion is not caused by either retinal adaptation or lightness persistence. 


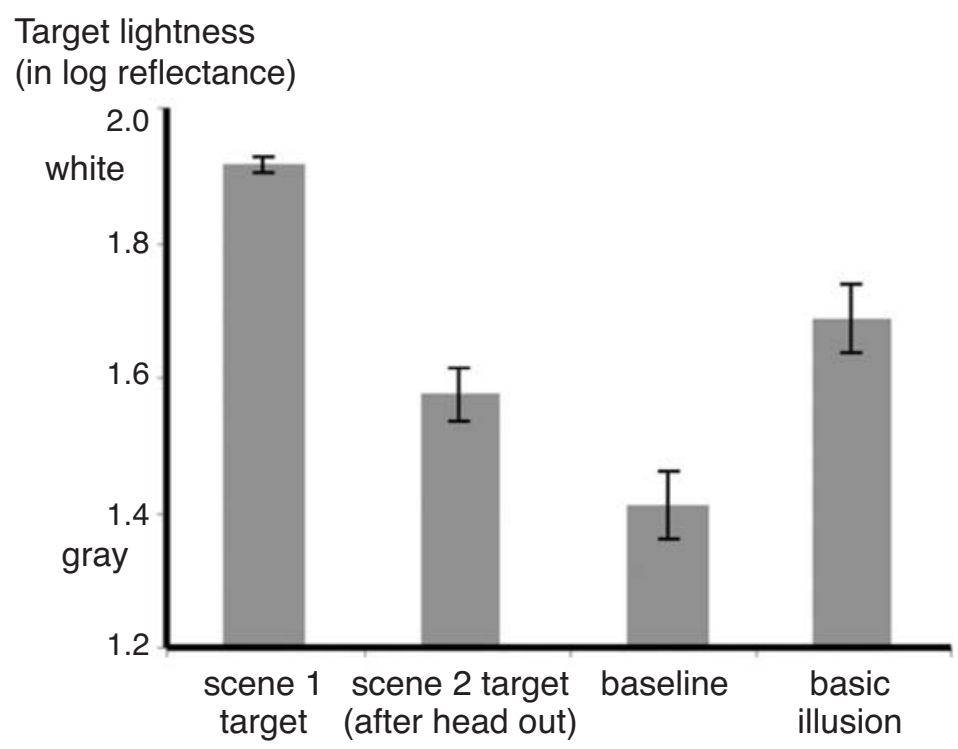

Figure 9. Results from Experiment 6, head removed from the Mondrian world between scenes. The graph shows that the lightness hangover is reduced but not completely eliminated when the observer looks out of the Mondrian viewing chamber between Scene 1 and Scene 2 .

When, in Experiment 2, we created conditions that, according to retinal adaptation, should have strengthened the illusion, we found that the illusion was completely eliminated. When, in Experiment 5, we created conditions that, according to lightness persistence, should have produced a strong illusion, we found no illusion.

It appears that our hangover illusion can be completely explained by anchor persistence. This resistance to the immediate application of the new anchor in Scene 2 is caused by the presence of a large number of continuously visible steady patches. Each such patch can be said to argue or "vote" against a change of illumination. Each continuously visible patch that maintains a constant luminance across scenes provides a small bit of information that the illumination has not changed.

Under normal conditions, the registration of a change in illumination is signaled by certain photometric changes that co-occur with the physical change in illumination: a shift in the anchor and a dynamic change in the luminance of individual surfaces. The detection of these changes produces a reanchoring of the scene. In our Mondrian world, however, we changed the highest luminance not by increasing illumination but by introducing surfaces of higher luminance. Thus the illumination change cues were de-coupled, producing conflicting information as to whether or not the illumination level had changed and, by implication, whether or not the scene must be reanchored. Whereas the upward shift in anchor suggested an increase in the illumination, the presence of the steady patches suggested no change.

If there was a change in illumination, as suggested by the upward shift in the anchor, then one or more of these patches would be expected to change in luminance and this would immediately attract attention. The more patches present in a scene, the greater the probability of dynamic change in these patches, if the illumination were to change. Thus the more the steady patches present in the scene, the greater the likelihood that the illumination level has not changed. To function in this way, steady patches need not be attended. But they must be continuously visible in order to exploit the passive monitoring capability of the visual system. When continuous visibility is disrupted, the effect of the steady patches is reduced (heads out) or eliminated (darkness interval).

It now appears likely that anchor persistence was at work in the results reported by Newson (1958), Cataliotti and Bonato (2003), and Wallach (1948). In all these cases, a target of constant luminance appeared to slowly change in lightness following a change of the highest luminance in the scene. The same may apply to Gilchrist (1980) as well, except that in this case there was no change in the highest luminance within the overall scene, but the target moved perceptually from a depth plane with one highest luminance to a depth plane with a different highest luminance.

The results of Shimozaki et al. (1999), on the other hand, may have been an example of lightness persistence. There, despite the phenomenal change in luminance of the target over time, the perceived change at Time 2 was always incomplete, seemingly restrained by the perceived lightness value at Time 1 .

Two important factors have not been completely explored in the present series of experiments. These are area effects and articulation. Several studies seem to suggest that the lightness of a surface is affected by its size ( $\mathrm{Li} \&$ 
Gilchrist, 1999; Gilchrist et al., 1999). It would be interesting to see whether larger (or smaller) steady patches would produce a change in the amount of hangover effect. One way in which this could be tested would be to use a larger Mondrian world.

Our results are closely related to the concept of articulation, which is generally understood to refer to the number of elements in a group. Katz (1935) found that the degree of lightness constancy for patches in a field of illumination depends on the extent of articulation within the field, specifically the number of visually distinct patches. Kardos (1934) showed that the lightness of a target is codetermined, partly in relation to its relevant field of illumination, and partly in relation to the foreign (usually neighboring) field. When the relevant field has a greater level of articulation, it is weighted more in the codetermination, leading to more constancy. More recent work done within the context of anchoring theory (Gilchrist et al., 1999) strongly suggests that articulation works by strengthening the framework to which the target belongs (for a full review, see Gilchrist \& Annan, 2002).

Perhaps a parallel analysis can be applied to our hangover illusion. Scene 1 and Scene 2 can be thought of as competing temporal fields of illumination or frameworks, like the spatial relevant and foreign fields of Kardos (1934). High articulation makes Scene 1 stronger in this competition. The steady patches belong to Scene 1 because that is where they first appear. This may make the Scene 1 anchor stronger, so that it is more resistant to change, even with the addition of a higher maximum luminance.

\section{REFERENCES}

Bolles, R. C., Hulicka, I. M., \& Hanly, B. (1959). Color judgments as a function of stimulus conditions and memory color. Canadian Journal of Psychology, 13, 175-178.

BRUner, J. S., Postman, L., \& Rodrigues, J. (1951). Expectation and the perception of color. American Journal of Psychology, 64, 216227.

BurZlafF, W. (1931). Methodologische Beiträge zum Problem der Farbenkonstanz [Methodological notes on the problem of color constancy]. Zeitschrift für Psychologie, 119, 117-235.

Cataliotti, J., \& Bonato, F. (2003). Spatial and temporal lightness anchoring. Visual Cognition, 10, 621-635.

Cataliotti, J., \& Gilchrist, A. [L.] (1995). Local and global processes in lightness perception. Perception \& Psychophysics, 57, $125-$ 135.

DAVIS, J. T., \& Brooks, C. I. (1977). Judged brightness as a function of dark, intermediate, and light adaptation. Perceptual \& Motor Skills, 44, 987-990.

DUNCKER, K. (1939). The influence of past experience upon perceptual properties. American Journal of Psychology, 52, 255-265.

Gelb, A. (1929). Die "Farbenkonstanz" der Sehdinge. In A. Bethe,
G. von Bergmann, G. Embden, \& A. Ellinger (Eds.), Handbuch der normalen und pathologischen Psychologie (Vol. 12, pp. 594-678). Berlin: Springer-Verlag.

GiLCHRIST, A. L. (1980). When does perceived lightness depend on perceived spatial arrangement? Perception \& Psychophysics, 28, 527538.

Gilchrist, A. L., \& AnnAN, V., JR. (2002). Articulation effects in lightness: Historical backgrounds and theoretical implications. Perception, 31, 141-150.

Gilchrist, A. [L.], Kossyfidis, C., Annan, V., Economou, E., Bonato, F., Agostini, T., Cataliotti, J., Li, X., \& Spehar, B. (1999). An anchoring theory of lightness perception. Psychological Review, 106, 795-834.

HANAWALt, N. G., \& Post, B. E. (1942). Memory trace for color. Journal of Experimental Psychology, 30, 216-227.

Helmholtz, H. vON (1962). Helmholtz's treatise on physiological optics (J. P. C. Southall, Ed. \& Trans.). New York: Dover. (Original work published 1866)

HERING, E. (1964). Outlines of a theory of the light sense (L. M. Hurvich \& D. Jameson, Trans.). Cambridge, MA: Harvard University Press. (Original work published 1874)

Hurvich, L. M., \& JAMESON, D. (1966). The perception of brightness and darkness. Boston: Allyn \& Bacon.

Kardos, L. (1934). Ding und Schatten: Eine experimentelle Untersuchung über die Grundlagen des Farbensehens [Thing and shadow: An experimental investigation on the foundations of color vision]. Zeitschrift für Psychologie, 23(Suppl.).

Katz, D. (1935). The world of colour. London: Kegan Paul, Trench, Trubner.

Klein, R., Kingstone, A., \& Pontefract, A. (1992). Orienting of visual attention. In K. Rayner (Ed.), Eye movements and visual cognition: Scene perception and reading (pp. 46-65). New York: SpringerVerlag.

KöHLER, W. (1917). Die Farbe der Sehdinge beim Schimpansen und beim Haushuhn [The color of visually perceived objects in the chimpanzee and domestic fowl]. Zeitschrift für Psychologie, 77, 248-255.

KREIS, J. vON (1905). Die Gesichtsempfindungen [The sense of vision]. In W. A. Nagel (Ed.), Handbuch der Physiologie des Menschen: 3 Bd. Physiologie der Sinne (pp. 109-282). Braunschweig: Vieweg.

Li, X., \& GiLchrist, A. L. (1999). Relative area and relative luminance combine to anchor surface lightness values. Perception \& Psychophysics, 61, 771-785.

Newson, L. J. (1958). Some principles governing changes in the apparent lightness of test surfaces isolated from their normal backgrounds. Quarterly Journal of Experimental Psychology, 10, 82-95.

Over, R., Broerse, J., Crassini, B., \& Lovegrove, W. (1974). Orientation-specific aftereffects and illusions in the perception of brightness. Perception \& Psychophysics, 15, 53-56.

Shimozaki, S. S., Eckstein, M., \& Thomas, J. P. (1999). The maintenance of apparent luminance of an object. Journal of Experimental Psychology: Human Perception \& Performance, 25, 1433-1453.

TERNUS, J. (1938). The problem of phenomenal identity. In W. D. Ellis (Ed.), A source book of Gestalt psychology (pp. 149-160). New York: Harcourt, Brace.

WALLACH, H. (1948). Brightness constancy and the nature of achromatic colors. Journal of Experimental Psychology, 38, 310-324.

ZuCKERMAN, C. B., \& Rock, I. (1957). A reappraisal of the roles of past experience and innate organizing processes in visual perception. $P$ sychological Bulletin, 54, 269-296.

(Manuscript received December 19, 2002;

revision accepted for publication November 18, 2003.) 